\title{
Estructura de tamaño de la comunidad de macroinvertebrados acuáticos en un río de características semiáridas durante el estiaje (río Chícamo, S.E. España)
}

\author{
Cristina Guerrero*; Ma Rosario Vidal-Abarca* (1); Ma Luisa Suárez*; Rosa Gómez*; \\ Manuel Ortega**
}

* Departamento de Ecología e Hidrología. Universidad de Murcia. Campus de Espinardo. 30100 Murcia. (1) e-mail: charyvag@um.es

** Departamento de Biología Vegetal y Ecología. Universidad de Almería. Carretera de Sacramento s/n. La Cañada de San Urbano. 04120 Almería.

\section{RESUMEN}

En este trabajo se analiza la estructura de tamaño de la comunidad de macroinvertebrados acuáticos del río Chícamo, en términos de densidad y biomasa, durante el estiaje del verano de 1999. Los muestreos se realizaron cada dos semanas durante Julio y Agosto, con el fin de describir cómo la distribución de tamaños de la comunidad varía en el espacio (hábitats, definidos según el tipo de sustrato, concentración de fósforo reactivo soluble en el agua: PRS y biomasa de perifiton) y en el tiempo.

Aunque existen diferencias en la densidad de organismos entre las semanas de muestreo, la forma del espectro de tamaño fue similar a lo largo del estudio, existiendo mayores diferencias espaciales, fundamentalmente en la densidad y distribución de los organismos de mayor tamaño.

Los dípteros dominan numéricamente en el río Chícamo, mientras que los individuos de mayor tamaño, pertenecientes a los coleópteros, son los que aportan mayor biomasa al sistema. La concentración de PRS y la biomasa de perifiton, no influyeron en la densidad ni en la distribución de organismos por clase de tamaño, lo que indica que no actúan como factores limitantes para la producción secundaria en el tramo de estudio, al igual que ocurre con el tipo de sustrato.

La no inclusión de la meiofauna en el estudio, y el tamaño de luz de malla $(250 \mu \mathrm{m})$ empleado en la recogida de los organismos, determinaron probablemente que la forma del espectro de tamaño obtenido se aleje de las curvas unimodales obtenidas para otros sistemas lóticos.

Palabras clave: espectro de tamaño; biomasa; densidad; macroinvertebrados acuáticos; río semiárido; sequía.

\section{ABSTRACT}

We analyzed the size spectra (biomass and density) of macroinvertebrate community in the Chicamo stream during the summer drought of 1999. During July and August we collected samples of aquatic macroinvertebrates every two weeks and from different habitats with different substrate types, allowing assessment of temporal and spatial variation. Moreover, we measured SRP (soluble reactive phosphorus), and periphyton biomass to detect the influences of them on macroinvertebrate density.

No changes in form of size spectra were detected during the study period in spite of differences in macroinvertebrate density among sampling dates. Spatial differences were higher than temporal ones.

Diptera are dominant in the aquatic community of the Chicamo stream, but the beetles contributed with higher values of biomass. We have not found any relationship between substrate types, SRP, or periphyton biomass and density or spatial distribution of macroinvertebrates in each size class. So, these variables seems not to be limiting factors for secondary productivity in Chicamo stream.

The differences observed in uni-modal curves of size spectra in relation to other aquatic ecosystems may be due to the exclusion of meiofauna as a consequence of the mesh used to collect macroinvertebrates (250-um).

Keywords: size-spectra; biomass; density; aquatic macroinvertebrates; semiarid stream; drought. 


\section{INTRODUCCIÓN}

La estructura de las comunidades naturales se ha descrito generalmente utilizando la composición y la abundancia de las especies que la componen. Una alternativa a esta descripción más tradicional, es la utilización de la estructura de tamaño o espectro de tamaño (Sheldon et al., 1972), que presenta la ventaja de eliminar el primer paso en todo estudio de una comunidad: la identificación de las especies que la componen, siendo así útil para el análisis de las comunidades y los ecosistemas, al simplificar las comparaciones entre comunidades formadas por diferentes especies (Hanson et al., 1989). Aunque es relativamente fácil encontrar estudios sobre el espectro de tamaño en comunidades pelágicas y bentónicas marinas (p.e. Sheldon et al., 1972) y lacustres (Sprules et al., 1983; Strayer, 1986), apenas existen estudios para las comunidades que habitan los sistemas de aguas corrientes (Morin \& Nadon, 1991), y los que hay han sido llevados a cabo fundamentalmente en Estados Unidos y Canadá.

La estructura de tamaño de las comunidades acuáticas, está recibiendo nuevamente atención por parte de los ecólogos, dado que procesos fisiológicos como la respiración o la producción de comunidades enteras, y las interacciones entre los animales bentónicos y la estructura física del ambiente que les rodea, estan íntimamente relacionadas con el tamaño de cuerpo de las diferentes especies que la componen (Strayer, 1991; Poff et al., 1993; Morin \& Dumont, 1994).

Los escasos estudios realizados en sistemas lóticos demuestran que el espectro de tamaño de los invertebrados presenta una regularidad importante en el espacio y en el tiempo (Morin, 1997), al contrario de lo que ocurre con los principales descriptores de las comunidades biológicas, como la diversidad, abundancia, biomasa y producción, relacionado con la variabilidad espacio-temporal de los factores abióticos (Downes et al., 1993; Palmer \& Poff, 1997; Palmer et al., 1997). Así, el espectro de tamaño no varía entre ríos (Morin \& Nadon, 1991), sustratos (Bourassa \& Morin, 1995) y gradientes en las concentraciones de fósforo o en relación con la biomasa de perifiton (Bourasa \& Morin, 1995).

Aunque este tipo de estudios debe realizarse generalmente a lo largo de un ciclo hidrológico completo como mínimo, Morín et al. (1995) apuntan que la distribución media de tamaños varía poco a lo largo del ciclo anual, aún cuando se ve afectada por el reclutamiento de organismos de pequeño tamaño durante los meses de verano.

Los objetivos planteados en este trabajo son, por un lado, describir la estructura de tamaño de la comunidad de macroinvertebrados acuáticos del río Chícamo durante el verano de 1999 y por otro, analizar la variación espacial y temporal de dicha estructura con el fin de detectar los posibles efectos del estiaje. Además, se pretende analizar el efecto del tipo de sustrato, la concentración de fósforo reactivo soluble en el agua (PRS) y la biomasa de perifiton, como factores ambientales, en el espectro de tamaño obtenido. La selección del PRS obedece a que es el nutriente limitante en el río Chícamo (VidalAbarca et al., 2000), de manera que podría estar limitando la producción primaria del sistema y, en consecuencia también la secundaria.

En relación al sustrato, muchos estudios ponen de manifiesto su importancia en la distribución de los invertebrados acuáticos (Cummins, 1966; Cummins \& Lauff, 1969; Sheldon \& Haick, 1981). Sin embargo, no existen evidencias claras en cuanto a sus posibles efectos en la distribución de tamaños de estas comunidades (Strayer, 1986; Warwick \& Joint, 1987; Bourassa \& Morin, 1995; Solimini et al., 2001).

Por último, la biomasa de perifiton se ha seleccionado por tratarse del principal productor primario del sistema (Velasco, et al., 2003), constituyendo, junto con la materia orgánica bentónica (Martínez et al., 1998), la base de la cadena alimenticia en el tramo de estudio. Se trata, al igual que ocurre con el sustrato, de uno de los principales factores que influyen en la microdistribución de los organismos en sistemas lóticos (Egglishaw, 1964; Wallace \& Merrit, 1980; Sheldon \& Haick, 1981). 


\section{AREA DE ESTUDIO}

El río Chícamo, afluente del río Segura por su margen izquierda, se localiza en la Región de Murcia (Fig. 1). Presenta una longitud de 59.4 $\mathrm{Km}$, de los que únicamente 10 mantienen agua de forma permanente, por lo que se trata de un río espacialmente intermitente.

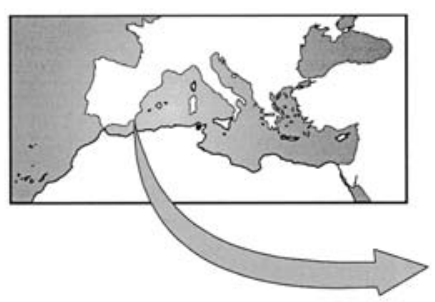

Cuenca del río Chícamo

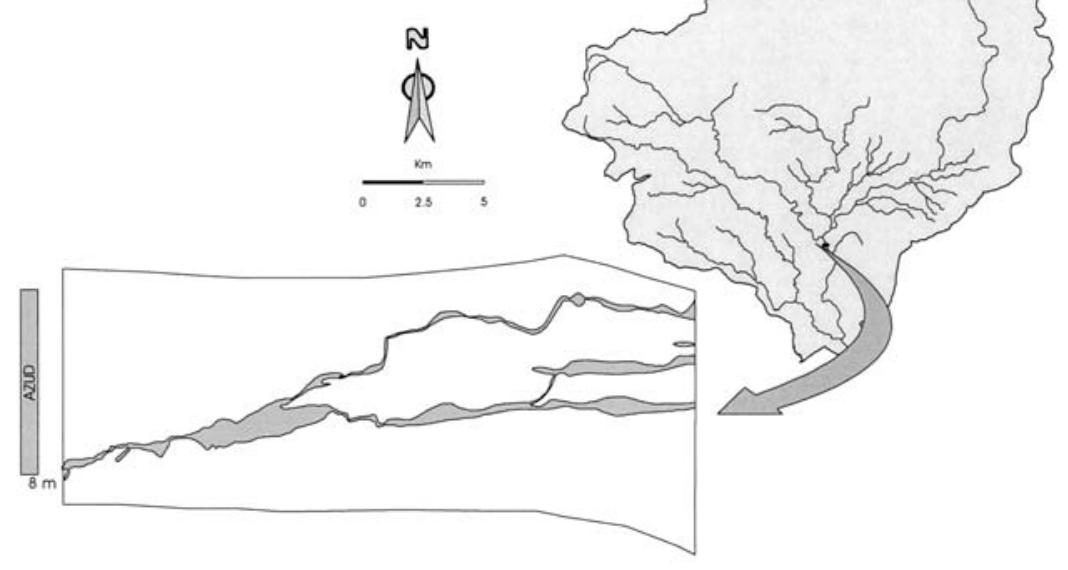

(1999) Abanilla C.H. $222 \mathrm{msnm}$
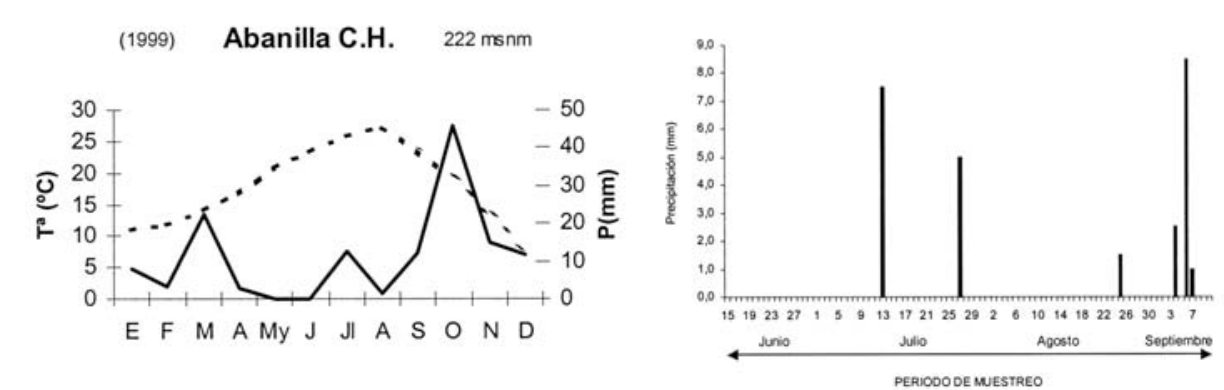

Figura 1. Localización geográfica de la cuenca del río Chícamo, esquema general del tramo de estudio, climodiagrama obtenido con datos meteorológico de la estación de Abanilla y precipitaciones diarias durante el período de muestreo. Geographic location of the Chicamo stream watershed, schematic representation of the studied section, weather diagram according with meteorological data from the Abanilla estation, and daily precipitation during the sampling period.
El rasgo climático más característico de la zona es la marcada irregularidad del régimen de precipitaciones, cuya manifestación extrema son los episodios de tipo torrencial. Estos fenómenos determinan una forma de cauces de gran anchura en relación con el caudal circulante, desprovistos prácticamente de vegetación de ribera.

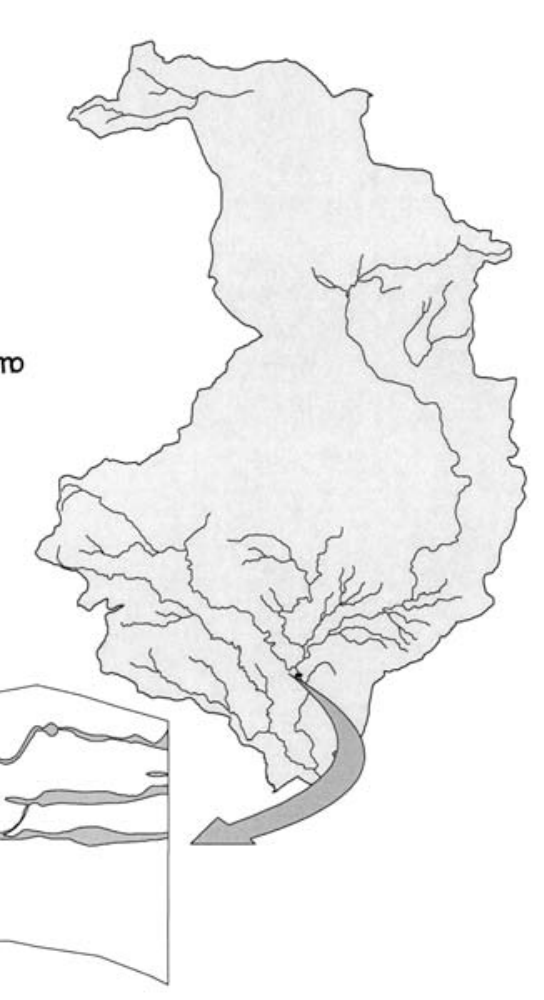


Tabla 1. Características de los diferentes hábitats muestreados en el río Chícamo durante el periodo de estudio. (Habitat corriente: velocidad de corriente $>0.05 \mathrm{~m} / \mathrm{s}$. Habitat poza: profundidad del agua $>4 \mathrm{~cm}$. Habitat somero: profundidad del agua $<4 \mathrm{~cm}$ ). Characteristics of the different habitats sampled in the Chicamo stream during the study period. (Current habitat: current velocity $>0.05 \mathrm{~m} / \mathrm{s}$. Pool habitat: water depth $>4 \mathrm{~cm}$. Shallow habitat: water depth $<4 \mathrm{~cm}$ ).

\begin{tabular}{clccc}
\hline HABITAT TIPO & AMBIENTE & SUSTRATO & VEGETACIÓN & PRESENCIA Fe $^{+++}$ \\
\hline HT1 & Corriente & Cantos & No & No \\
HT2 & Somero & Sedimento & Perifiton & No \\
HT3 & Profundo & Sedimento & Perifiton & No \\
HT4 & Profundo & Sedimento & Chara vulgaris & No \\
HT5 & Corriente & Grava+sedimento & Perifiton & No \\
HT6 & Profundo & Sedimento & Chara vulgaris + Juncus maritimus & No \\
HT7 & Profundo & Sedimento & Perifiton & No \\
HT8 & Corriente & Margas & No & No \\
HT9 & Profundo & Sedimento & Perifiton + Juncus maritimus & No \\
HT10 & Somero & Sedimento & Perifiton + Juncus maritimus & No \\
HT11 & Corriente & Cantos & Perifiton & No \\
HT12 & Profundo & Sedimento & Perifiton+J. maritimus + Phragmites australis & . \\
\hline
\end{tabular}

Para llevar a cabo el estudio se seleccionó un tramo de $100 \mathrm{~m}$, en el sector medio del río Chícamo donde el agua fluye de forma permanente que sufre un fuerte estiaje estival. El inicio del tramo lo constituye un pequeño azud totalmente colmatado, bajo el cual afloran las aguas subsuperficiales que circulan por el cauce (Fig. 1). El lecho del cauce está constituido por margas de naturaleza impermeable, sobre las que se depositan fundamentalmente cantos y gravas en zonas erosionales, existiendo un claro predominio de los sedimentos orgánicos en las áreas deposicionales.

La comunidad de macrófitos está constituida únicamente por manchas aisladas del género Chara, siendo el perifiton el principal productor primario del sistema (Suárez \& Vidal-Abarca, 2000; Velasco et al., 2003), cuya descomposición origina la fracción fina (dominante) de materia orgánica en el tramo de estudio (Martínez et al., 1998). La vegetación de ribera está representada por Phragmites australis, Tamarix canariensis, Juncus maritimus y Limonium sp., que se presentan de forma aislada en ambas márgenes del río.

Durante el periodo de estudio, la profundidad de la lámina de agua oscilo entre 8 y $0.3 \mathrm{~cm}$ y la velocidad de corriente nunca superó los $2 \mathrm{~m} / \mathrm{s}$. El estiaje supuso una reducción del $83.7 \%$ de la superficie total inicial de la lámina de agua (Guerrero, 2002).

\section{METODOLOGÍA}

\section{Programa de muestreo}

El programa de muestreo se desarrolló entre el 7 de julio y el 1 de septiembre de 1999, coincidiendo con las precipitaciones caídas la primera semana de septiembre. El proceso de desecación se vio interrumpido en dos ocasiones por las precipitaciones del 13 y del 27 de julio (Fig. 1). Dadas las escasas dimensiones de la lámina de agua, y con el fin de perturbar el sistema lo menos posible, la periodicidad del muestreo fue quincenal. Con el fin de recoger la máxima heterogeneidad espacial en el tramo de estudio, se seleccionaron un total de 12 habitats diferentes definidos en función de cuatro grupo de variables (Tabla 1): microambiente (corriente, somero y profundo); tipo de sustrato (cantos, gravas, sedimentos orgánicos y combinaciones de ellos), tipo de vegetación (perifiton, helófitos, sin vegeta- 
Tabla 2. Fechas y hábitats muestreados en el río Chícamo. Data and habitats sampled in the Chicamo stream.

\begin{tabular}{cc}
\hline Fecha & Hábitats \\
\hline $7 / 07 / 99$ & HT1, HT2, HT3, HT4, HT5, HT6, HT7, HT8, HT9 \\
$22 / 07 / 99$ & HT2, HT3, HT4, HT5, HT9, HT6, HT10, HT11 \\
$3 / 08 / 99$ & HT2, HT3, HT5, HT9, HT11 \\
$17 / 08 / 99$ & HT2, HT3, HT5, HT10, HT11 \\
$1 / 09 / 99$ & HT2, HT3, HT10, HT11, HT12 \\
\hline
\end{tabular}

ción y combinaciones de ellos) y presencia o ausencia de $\mathrm{Fe}^{+++}$en el sedimento (Guerrero, 2002). En la Tabla 2 se presentan las fechas de recogida de las muestras (físico-químicas y biológicas), y los hábitats muestreados. En total se obtuvieron 96 muestras.

\section{Recogida de datos físico-químicos}

En cada uno de los habitats muestreados se midió la velocidad de la corriente (molinete electromagnético M201D), anchura, longitud y profundidad de la lámina de agua, que posteriormente mediante el programa AUTOCAD 14, permitieron determinar la superficie del hábitat. Además, se tomaron datos de salinidad, conductividad (conductivímetro YSI-33), temperatura y concentración de oxígeno disuelto en el agua (oxímetro WTW, OXI 197-S). Durante gran parte del estudio, la recogida de agua para obtener la medida del fósforo reactivo soluble (PRS) (APHA, 1985) se realizó con una jeringuilla, dadas las escasas dimensiones de la lámina de agua.

Para calcular la biomasa de perifiton se utilizó un "core" de $2 \mathrm{~cm}$ de diámetro $\left(3.14 \mathrm{~cm}^{2}\right.$ de superficie) que era introducido a una profundidad de $2 \mathrm{~cm}$ en el sedimento. Las muestras obtenidas eran guardadas en una bolsa, y una vez en el laboratorio eran filtradas (filtros Whatman $\mathrm{CF} / \mathrm{C})$. A continuación los filtros se secaban en una estufa $\left(24 \mathrm{~h} \mathrm{a} 60^{\circ} \mathrm{C}\right)$, enfriados en el desecador y pesados con una precisión de $0.0001 \mathrm{~g}$ en una balanza Sartorius BP-221S. El peso seco libre de cenizas (PSLC) se obtuvo quemando en un horno mufla (4h a $550^{\circ} \mathrm{C}$ ), y calculando la diferencia entre peso seco (PS) y peso seco de las cenizas (PSC) (Aloi, 1990).

\section{Recogida y procesado de las muestras de macroinvertebrados acuáticos}

Para la recogida de los macroinvertebrados acuáticos, se utilizó un "core" de PVC de $13 \mathrm{~cm}$ de diámetro $\left(133 \mathrm{~cm}^{2}\right)$, siempre acoplado lo máximo posible al sustrato, de manera que tras removerlo, los organismos eran capturados con la ayuda de una bomba de vacío, y posteriormente filtrados con una manga de luz de malla de $250 \mu \mathrm{m}$. Todas las muestras fueron conservadas en alcohol al $70 \%$. Una vez en el laboratorio, los organismos eran lavados, separados e identificados al máximo nivel posible, contados y medidos, estableciéndose diferentes clases de tamaño en función del táxon analizado.

Para las medidas de longitud de los individuos, se tomó la distancia desde la parte anterior de la cabeza hasta el último segmento abdominal, excluyendo los cercos en el caso de efémeras, y larvas de coleópteros, y las branquias en el caso de los odonatos zigópteros. Para el crustaceo Echinogammarus veneris y las familias de dípteros Chironomidae, Ceratopogonidae y Ephydridae, dada la curvatura de sus cuerpos, la longitud se estimó estableciendo una línea recta desde la parte anterior ventral de la cabeza hasta el límite ventral posterior del último segmento abdominal (Burgherr \& Meyer, 1997). Las medidas de la longitud corporal se realizaron bajo lupa binocular, estableciéndose las diferentes clases de tamaño, con una precisión de $1 \mathrm{~mm}$. 
Una vez identificados y medidos, grupos de individuos pertenecientes al mismo táxon y clase de tamaño eran introducidos, en número variable en función del tamaño concreto, en una estufa $\left(24 \mathrm{~h}\right.$ a $\left.60^{\circ} \mathrm{C}\right)$, enfriados en un desecador (24 h) y pesadas en una balanza con precisión de $0.0001 \mathrm{~g}$ (Sartorius BP-221S) para obtener el peso seco (PS). Estas condiciones ayudan a prevenir las posibles pérdidas de biomasa por volatilización de los componentes orgánicos (Hynes, 1982).

Aunque se midieron todos los individuos presentes en las diferentes muestras, únicamente aquellos que presentaban intactas todas las estructuras corporales fueron seleccionados para el cálculo del peso seco. Para las estimas finales de PS, no se consideraron las posibles pérdidas debidas al uso del alcohol como medio de conservación de las muestras, ya que se asume que estas pérdidas no van a variar entre los diferentes muestreos y sitios muestreados (las muestras fueron separadas y pesadas entre cinco y seis meses después de su recogida) (Morin, 1991).

Algunos de los táxones no se tuvieron en cuenta para el cálculo de la biomasa debido a que el número de individuos existentes no era suficiente para realizar los cálculos por clase de tamaño (p.e. Caenis luctuosa, Dolicopodidae, Anthomyidae y Simuliidae). Para el resto, los cálculos de PS para cada clase de tamaño, se realizó un máximo de cinco veces y un mínimo de una, en función del número de organismos recogidos en el tramo de estudio. Las clases de tamaño más pequeñas plantearon mayores problemas, debido a que el número de individuos necesarios para poder realizar las estimas era mayor. Los resultados de densidad y biomasa se expresan en términos de $\mathrm{n}^{\circ} \mathrm{ind} / \mathrm{m}^{2}$ y g PS $/ \mathrm{m}^{2}$, respectivamente.

Para definir el grupo funcional al que pertenece cada táxon, se han utilizado las clasificaciones de Cummins (1973) y Tachet et al. (2000). Para las especies que no han podido ser identificadas, como los dípteros, dado que sí se conocen las subfamilias predominantes, se ha optado por dividir el número total de individuos en función del número de grupos funcionales a los que pue- den pertenecer. Del mismo modo para aquellas especies que pueden utilizar varios mecanismos de alimentación (p.e. Sigara scripta), se ha empleado el mismo procedimiento.

\section{Análisis estadístico de los datos}

Para el análisis del espectro de tamaño de la comunidad de macroinvertebrados acuáticos del río Chícamo, los organismos se agruparon en clases de tamaño logarítmico. Para su definición, y con el fin de establecer un número de intervalos de peso adecuado al número de muestras recogidas, se utilizó la fórmula de Sturges (1926), donde $\mathrm{n}$ es el número de muestras, I es el número de intervalos, y $\mathrm{E}$ es la función parte entera.

$$
\mathrm{I}=1+\mathrm{E}\left(3.32+\log _{10} 96\right)=7
$$

El número así obtenido, se incrementó en dos para contemplar las clases de tamaño que constituyen los límites inferior y superior, dado que en el primer caso la densidad de individuos es muy elevada, y en el último muy baja. Finalmente se definieron $9 \log _{10}$ clases de tamano que varían entre 2.7 y $2.510^{4} \mu \mathrm{g}$ PS.

Para examinar los efectos de la fecha de muestreo y el hábitat en la densidad de organismos por clase de tamaño, se consideró la densidad en cada clase de tamaño, como variable dependiente, y la fecha y el hábitat como variables independientes. Se ha utilizado un Modelo Lineal General de ANOVA (GLM, Neter et al., 1996), mediante el Método de Pasos Sucesivos, para determinar qué variables independientes son las que mejor explican la dependiente. El criterio utilizado es que la probabilidad de entrada de una variable es $\leq 0.05$, y la probabilidad de salida $\geq 0.10$.

Debido a que no todas las clases de tamaño presentan individuos en todas las fechas y en todos los hábitats, los datos de densidad fueron transformados previamente al análisis, mediante la expresión $\log _{10}($ densidad +3.14$)$, dado que 3.14 es la densidad mínima de individuos encontrada en una muestra. 
Tabla 3. Clases de tamaño ( en negrita) y biomasa media individual (gPS $/ \mathrm{m}^{2}$ ) de cada uno de las taxones considerados en el estudio. Los asteriscos señalan aquellos taxones que por el bajo número de individuos o por su mal estado de conservación, únicamente se han sometido al proceso de desecación en un caso $(*)$, en dos $(* *)$ o en tres $(* * *)$. En el resto de casos, el número de muestras analizadas ha sido de cinco. Size class (in bold) and individual mean biomass $\left(\mathrm{gPS} / \mathrm{m}^{2}\right)$ for each taxa considered in the study. Asteriscs designate those taxa for which, because of the low number of individuals or due to decomposition, the number of samples subjected to the dissecation process was only one (*), two $(* *)$, or three $(* * *)$. For the remainder taxa, the number of samples was five.

CLASES DE TAMAÑO (mm)

\begin{tabular}{|c|c|c|c|c|c|c|c|c|c|c|c|}
\hline INSECTOS & & & & & & & & & & & \\
\hline Dípteros & 15 & 10 & $>6$ & 6 & $4-2$ & $<2$ & & & & & \\
\hline Chironomidae & & & 0.172 & 0.071 & 0.025 & 0.005 & & & & & \\
\hline Ceratopogonidae & & & 0.063 & 0.041 & 0.013 & 0.003 & & & & & \\
\hline Ephydridae & & & 0.590 & 0.372 & 0.109 & & & & & & \\
\hline Tabanidae & 2 & 1 & & 0.236 & 0.039 & $0.011^{*}$ & & & & & \\
\hline Coleópteros & Adultos & Larvas $>6$ & 6-4 & $4-2$ & & & & & & & \\
\hline Nebrioporus baeticus & 2.023 & & & & & & & & & & \\
\hline Berosus hispanicus & 1.670 & & & & & & & & & & \\
\hline Larvas Berosus & & 1.362 & 1.249 & $0.057^{*}$ & & & & & & & \\
\hline Enochrus politus & 3.331 & & & & & & & & & & \\
\hline Enochrus falcarius & 2.213 & & & & & & & & & & \\
\hline Larvas Enochrus & & 0.466 & 0.236 & $0.065^{*}$ & & & & & & & \\
\hline Ochthebius cuprescens & 0.201 & & & & & & & & & & \\
\hline O. delgadoi & 0.172 & & & & & & & & & & \\
\hline O. tacapasensis & 0.081 & & & & & & & & & & \\
\hline O. tudmirensis & $0.273^{* *}$ & & & & & & & & & & \\
\hline Laccobius moraguesi & 0.736 & & & & & & & & & & \\
\hline Heterópteros & 9 & 7 & $>4$ & $4-2$ & $<2$ & & & & & & \\
\hline Sigara scripta & & & 0.953 & 0.264 & 0.101 & & & & & & \\
\hline Micronecta scholtzi & & & & $0.116^{*}$ & $0.027 *$ & & & & & & \\
\hline Naucoris maculatus & 15.580 & $9.467 * * *$ & & & & & & & & & \\
\hline Odonatos & 17 & 15 & 12 & 11 & 10 & 9 & 8 & 7 & 6-4 & $4-2$ & $<2$ \\
\hline Orthetrum brunneum & 25 & $20.050^{* *}$ & 15 & 9 & 5 & 5 & $3.333^{* * *}$ & 2 & 1 & 0 & $0.020^{*}$ \\
\hline Ichnura elegans & & & & & 3 & 1 & 1 & & 0 & 0 & \\
\hline Anax parthenope & & & & & & & $0.767 *$ & & & & \\
\hline Efemerópteros & $>6$ & $6-4$ & $4-2$ & $<2$ & & & & & & & \\
\hline Cloeon inscriptum & 1.074 & 0.502 & 0.240 & 0.011 & & & & & & & \\
\hline NO INSECTOS & & & & & & & & & & & \\
\hline Crustáceos & $>0.7$ & $0.7-0.5$ & $0.5-0.3$ & $<0.3$ & & & & & & & \\
\hline Echinogammarus veneris & 2.117 & 0.791 & 0.409 & 0.071 & & & & & & & \\
\hline Oligoquetos & $>6$ & $6-4$ & $<4$ & & & & & & & & \\
\hline Oligochaeta sp. & 0.086 & 0.045 & 0.019 & & & & & & & & \\
\hline Hidrácaros & $>2$ & $<2$ & & & & & & & & & \\
\hline Hidracarina sp. & 0.536 & 0.233 & & & & & & & & & \\
\hline
\end{tabular}

Se transformaron todas las variables independientes a formas logarítmicas para linealizar las relaciones con la variable dependiente, y reducir los valores extremos en el coeficiente de determinación, y a incluir términos de orden mayor en el caso del peso, con el fin de tener en cuenta la curvilinealidad. De esta manera, se introdujeron en el modelo los términos lineal, cuadradro, cúbico, cuádruple y quíntuple del peso, aunque únicamente se mantuvieron los términos lineal y cuadrado, ya que eran los que reducían de forma significativa la varianza residual.

Con el fin de detectar los posibles efectos del tamaño del sustrato, la biomasa de perifiton, la concentración de PRS y el tipo de hábitat, sobre la densidad de organismos por clase de tamaño, 
se utilizó un Modelo Lineal General, en el que la densidad de organismos (densidad +3.14 ) fue la variable dependiente. El sustrato fue incluido en el análisis como factor fijo, mientras que la biomasa de perifiton, la concentración de fósforo reactivo soluble en el agua y los términos lineal y cuadrático del peso, se incluyeron como covariables. En este segundo análisis únicamente se tuvieron en cuenta las cuatro últimas prospecciones, debido a la falta de datos de PRS y biomasa de perifiton del 7/7/99.

\section{RESULTADOS}

\section{Densidad y biomasa de la comunidad de macroinvertebrados acuáticos}

En la Tabla 3, se presentan las clases de tamaño y los valores de biomasa media individual, para cada uno de los taxones considerados en el estudio. En total se contaron, midieron y calculó la biomasa de 32918 individuos de los 33612 recogidos durante las cinco semanas de muestreo.

En la Tabla 4, se presentan los valores totales y medios del $\mathrm{n}^{\mathrm{o}}$ de individuos, densidad, y biomasa para cada una de las prospecciones realizadas. En cuanto a los valores de densidad, existe una clara diferencia entre los alcanzados por los dípteros $\left(19740 \mathrm{ind} / \mathrm{m}^{2}\right)$ y el resto de los grupos. Los oligoquetos, segundo grupo en importancia, presentaron un valor medio de $2019 \mathrm{ind} / \mathrm{m}^{2}$. Entre los dípteros (Tabla 5), la familia Ceratopogonidae es la que presentó los valores más elevados de densidad para el estu- dio (11144 ind $\left./ \mathrm{m}^{2}\right)$. Entre los coleópteros, Ochthebius cuprescens es el que alcanzó mayores densidades $\left(741 \mathrm{ind} / \mathrm{m}^{2}\right)$, mientras que entre los heterópteros fue Sigara scripta (610 ind $/ \mathrm{m}^{2}$ ), y para los odonatos, Orthetrum brunneum $\left(123 \mathrm{ind} / \mathrm{m}^{2}\right)$.

La biomasa media de la comunidad, fue de $2.31 \mathrm{~g} \mathrm{PS} / \mathrm{m}^{2}$ para el conjunto de las prospecciones. El valor máximo se registró el 1/9/99 (2.766 g PS $\left./ \mathrm{m}^{2}\right)$, coincidiendo con el valor mínimo de inundación, el 3/8/99 (1.422 g $\mathrm{PS} / \mathrm{m}^{2}$ ) después de las precipitaciones que aterraron buena parte del cauce.

Los insectos contribuyeron a la biomasa media del tramo de estudio con $1.908 \mathrm{~g} \mathrm{PS} / \mathrm{m}^{2}$ (82.6\%), siendo la aportación del resto de los grupos (crustáceos, oligoquetos y ácaros) del $17.4 \%$ (0.402 g PS $\left./ \mathrm{m}^{2}\right)$. Entre los órdenes de insectos, destacan los coleópteros, con una biomasa media de $0.998 \mathrm{~g} \mathrm{PS} / \mathrm{m}^{2}$ (43.2\%), seguidos por dípteros $(0.332,14.4 \%)$, odonatos $(0.305,13.2 \%)$, heterópteros $(0.241,10.4 \%)$ y efemerópteros $(0.032,1.4 \%)$. Dentro del grupo de los no insectos destacan los crustáceos con una biomasa media de $0.329 \mathrm{~g} \mathrm{PS} / \mathrm{m}^{2}(14.2 \%)$ seguidos por oligoquetos $(0.065,2.8 \%)$ y ácaros $(0.008,0.4 \%)$.

Cuando se analizan las contribuciones de los diferentes taxones a la biomasa obtenida en las distintas semanas de muestreo (Tabla 5), se observa que Nebrioporus baeticus, Orthetrum brunneum, Sigara scripta y la familia Chironomidae, dentro de los insectos, y Echinogammarus veneris, entre los no insectos, son los que presentaron los mayores valores. Por

Tabla 4. Valores totales y medios del $\mathrm{n}^{\circ}$ de individuos ( $\mathrm{N}^{\mathrm{o}}$ ind), densidad (ind $\left./ \mathrm{m}^{2}\right)$ y biomasa $\left(\mathrm{g} \mathrm{PS} / \mathrm{m}^{2}\right)$ de la comunidad de macroinvertebrados acuáticos del río Chícamo, durante el periodo de estudio. Total and mean values of the number of individuals $\left(n^{\circ}\right.$ ind), density (ind $\left./ m^{2}\right)$, and biomass ( $g$ dry weight $/ \mathrm{m}^{2}$ ) of the aquatic macroinvertebrate community in the Chicamo stream during the study period.

\begin{tabular}{lccccc}
\hline Fecha & $\begin{array}{c}\text { Superficie } \\
\text { muestreada }\left(\mathrm{m}^{2}\right)\end{array}$ & $\mathrm{N}^{\mathrm{o}}$ muestras & $\mathrm{N}^{\mathrm{o}}$ Ind & Densidad (ind $\left./ \mathrm{m}^{2}\right)$ & Biomasa $\left(\mathrm{gPS} / \mathrm{m}^{2}\right)$ \\
\hline $7 / 7 / 99$ & 0.358 & 27 & 7916 & 22100 & 2.735 \\
$22 / 7 / 99$ & 0.318 & 24 & 11490 & 36087 & 1.969 \\
$3 / 8 / 99$ & 0.199 & 15 & 3107 & 15613 & 1.422 \\
$17 / 8 / 99$ & 0.199 & 15 & 6338 & 31850 & 2.660 \\
$1 / 9 / 99$ & 0.199 & 15 & 4067 & 20437 & 2.766 \\
\hline
\end{tabular}


Tabla 5. Aportación de los diferentes taxones que componen la comunidad de macroinvertebrados acuáticos del río Chícamo, a la densidad y biomasa, total y media, registrada durante el periodo de estudio. Contribution of the different taxa of the macroinvertebrate community in the Chicamo stream, to the density and biomass, total and mean,recorded during the study period.

\begin{tabular}{|c|c|c|c|}
\hline & & Densidad (ind/m²) & Biomasa $\left(\mathrm{gPS} / \mathrm{m}^{2}\right)$ \\
\hline \multirow{27}{*}{ 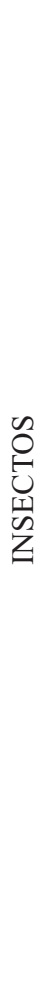 } & Dípteros & & \\
\hline & Quironómidos & 8383 & 0.156 \\
\hline & Ceratopogónidos & 11144 & 0.137 \\
\hline & Efídridos & 38 & 0.009 \\
\hline & Tabánidos & 175 & 0.030 \\
\hline & Coleópteros & & \\
\hline & Nebrioporus baeticus & 271 & 0.549 \\
\hline & Berosus hispanicus & 46 & 0.077 \\
\hline & Larvas Berosus & 17 & 0.008 \\
\hline & Enochrus politus & 20 & 0.066 \\
\hline & Enochrus falcarius & 18 & 0.040 \\
\hline & Larvas Ennochrus & 61 & 0.012 \\
\hline & Ochthebius cuprescens & 741 & 0.149 \\
\hline & O. delgadoi & 368 & 0.063 \\
\hline & O. tacapasensis & 126 & 0.010 \\
\hline & O. tudmirensis & 7 & 0.002 \\
\hline & Laccobius moraguesi & 30 & 0.022 \\
\hline & Heterópteros & & \\
\hline & Sigara scripta & 610 & 0.219 \\
\hline & Micronecta scholtzi & 92 & 0.006 \\
\hline & Naucoris maculatus & 1 & 0.016 \\
\hline & Odonatos & & \\
\hline & Orthetrum brunneum & 123 & 0.287 \\
\hline & Ichnura elegans & 34 & 0.013 \\
\hline & Anax parthenope & 6 & 0.005 \\
\hline & Efemerópteros & & \\
\hline & Cloeon inscriptum & 97 & 0.032 \\
\hline \multirow{5}{*}{$\begin{array}{l}n \\
0 \\
0 \\
0 \\
I \\
\tilde{Z} \\
Z \\
0 \\
Z\end{array}$} & Crustaceos & & \\
\hline & Echinogammarus veneris & 766 & 0.329 \\
\hline & Oligoquetos & & \\
\hline & Oligoquetos sp. & 2019 & 0.065 \\
\hline & $\begin{array}{l}\text { Hidracaros } \\
\text { Hidrácaros sp. }\end{array}$ & 24 & 0.008 \\
\hline
\end{tabular}

el contrario, Ochthebius tudmirensis, Anax parthenope, Micronecta scholtzi, las larvas de Berosus hispanicus y la familia Ephydridae, entre los insectos, y la familia Hydracarina, entre los no insectos, son los que presentan los valores mínimos de biomasa para el periodo de estudio.

En el análisis de los valores de densidad y biomasa en función de los diferentes grupos funcionales se observa (Fig. 2a) que el grupo de "colectores-raspadores" (collector-browsers, Tachet et al., 2000) exhibió los valores más ele- vados de densidad, mientras que para la biomasa (Fig. 2b), fueron los depredadores los de mayor aportación a la comunidad. En general, se observó un incremento en la densidad de los depredadores a lo largo de periodo de muestreo hasta alcanzar un máximo el 17/8/99 (12261 ind $\left./ \mathrm{m}^{2}\right)$. Ese pico coincide con el alcanzado por los filtradores (3251 ind $\left./ \mathrm{m}^{2}\right)$, mientras que los colectores-raspadores alcanzaron su máxima densidad el 22/7/99 (21838 ind/ $\mathrm{m}^{2}$ ), después de las precipitaciones de la semana anterior (Fig. 

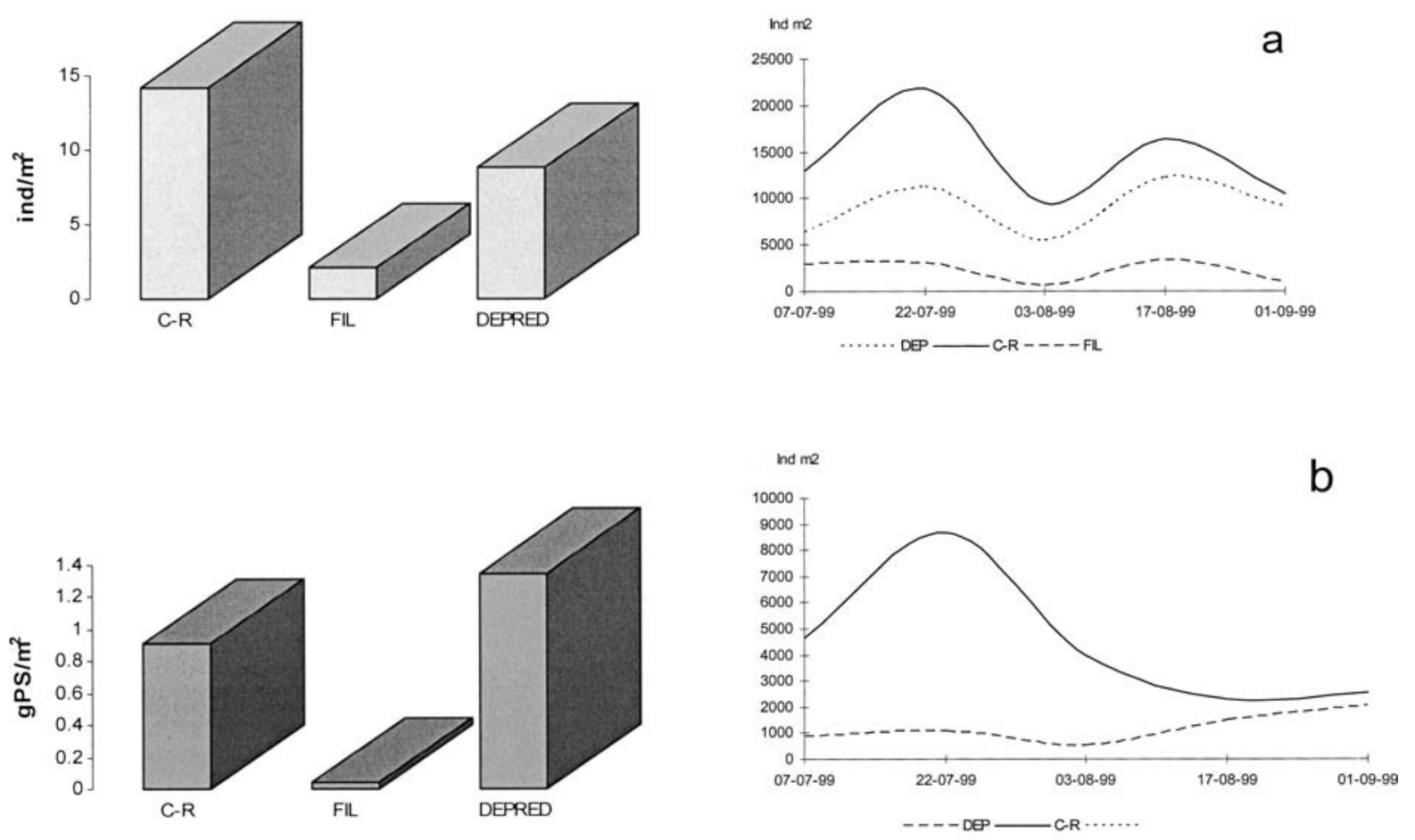

Figura 2. a) Densidad (ind $/ \mathrm{m}^{2}$ ) y b) Biomasa $\left(\mathrm{gPS} / \mathrm{m}^{2}\right)$ de cada uno de los grupos tróficos a los que pertenecen los diferentes taxones que componen la comunidad de macroinvertebrados acuáticos del río Chícamo. C-R: Colectores-raspadores; FIL: Filtradores; DEP: Depredadores (según Tachet et al., 2000). a) Density $\left(\mathrm{ind} / \mathrm{m}^{2}\right)$ and b) Biomass $\left(\mathrm{gDW} / \mathrm{m}^{2}\right)$ of each one of the trophic groups of the aquatic macroinvertebrate community in the Chicamo stream. C-R: Collectors-browsers; FIL: filterers; DEP: Predators (According with Tachet et al., 2000).

3a). Si el análisis se realiza obviando las aportaciones de los dípteros (Fig. 3b), fundamentalmente quironómidos y ceratopogónidos, se observa un patrón muy diferente. La abundancia y por tanto la densidad de los depredadores aumenta paulatinamente, a excepción de la semana posterior a las precipitaciones (3/8/99), hasta alcanzar un máximo la última semana de muestreo (2063 ind $\left./ \mathrm{m}^{2}\right)$, mientras que el grupo de los colectores-raspadores disminuye paulatinamente su densidad, hasta alcanzar un valor de $2565 \mathrm{ind} / \mathrm{m}^{2}$ la última semana de muestreo. Aunque en ningún momento la densidad de los depredadores supera la de los colectores-raspadores, al final del ciclo analizado, este grupo funcional perdió más del $70 \%$ de los organismos presentes al comienzo del estudio.

Figura 3. Evolución temporal de la densidad de organismos por grupo trófico para cada una de las prospecciones realizadas. a) incluyendo a los dípteros y b) excluyendo a los dípteros. C-R: Colectores-raspadores; FIL: Filtradores; DEP: Depredadores. Temporal evolution of the trophic groups' density of organisms for each sample date. a) includes dipterans b) excludes dipterans. C-R: Browsers; FIL: Filterers; DEP: Predators.

\section{Estructura de tamaño de la comunidad de macroinvertebrados acuáticos en el tramo de estudio}

En la figura 4a, se presenta el espectro de tamaño de la comunidad de macroinvertebrados acuáticos del río Chícamo durante el verano de 1999, para el total de las 96 muestras. Los individuos comprendidos entre 0.9 y 1.4 , es decir entre 10 y $20 \mu \mathrm{g}$ PS, son los que presentan mayores densidades durante todo el periodo de estudio. Por el contrario, los de mayor tamaño, entre 1.5 y $2510^{3} \mu \mathrm{g}$ PS (entre 3.2 y $4.2 \log 10$ ) presentan las menores densidades. Cuando se analiza la forma del espectro de tamaño en función de la biomasa de los taxones que componen la comunidad, el patrón es claramente dife- 

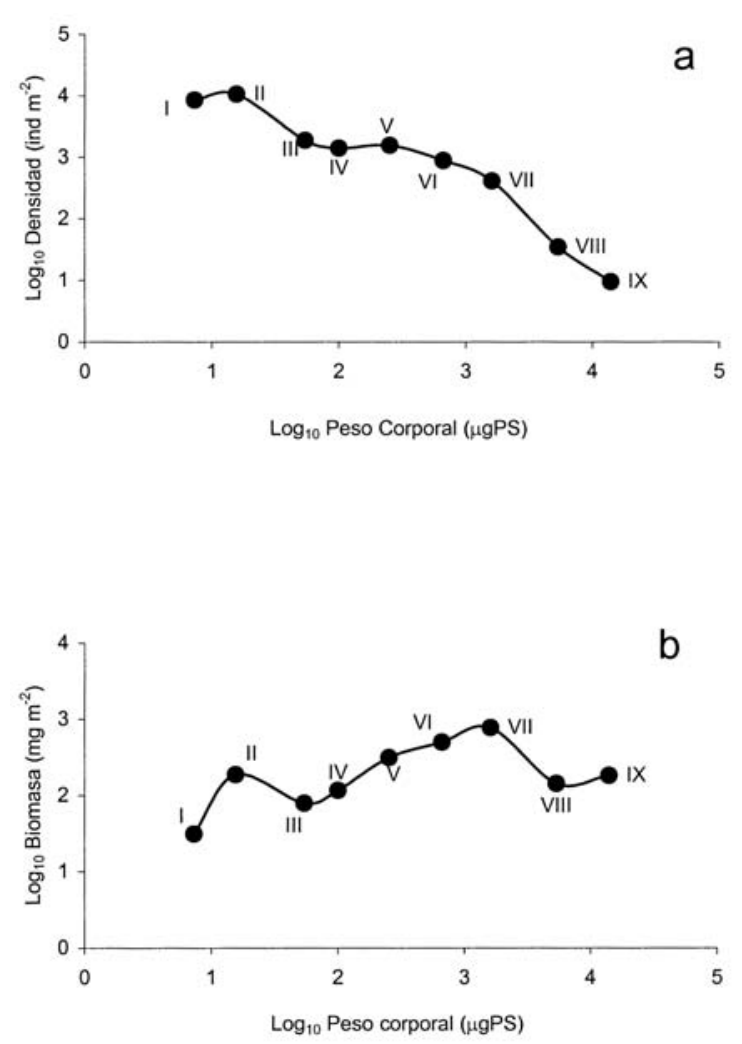

Figura 4. Espectro de tamaño medio, en términos de densidad (a) y biomasa (b), de la comunidad de macroinvertebrados acuáticos del río Chícamo. Los puntos son la densidad media para cada clase de tamaño $(\mathrm{n}=96)$. Mean size spectra expressed as density (a) and biomass (b) of the macroinvertebrate community in the Chicamo stream. Points are mean density for each size class $(n=96)$. b).

rente (Fig. 4b). En este caso, se obtiene una curva bimodal, con un primer pico correspondiente a los organismos con pesos comprendidos entre 10-20 $\mu \mathrm{g}$ PS, y un segundo para aquellos entre 1-3 mg PS, siendo éstos los que aportan mayor biomasa al sistema.

Tanto la densidad de macroinvertebrados como la biomasa por clase de tamaño en las diferentes semanas de muestreo, parecen ser más variables que la propia forma de la estructura obtenida en los espectros generales. En cuanto a la densidad (Fig. 5), la clase II (clase II 10-20 $\mu \mathrm{g}$ ) se presenta como dominante en tres de las cinco semanas. Lo mismo ocurre con la biomasa de los individuos de la clase VII (1-3 $\mathrm{mg})$, que presenta mayor biomasa en tres de las cinco prospecciones. Cabe destacar la influencia de las precipitaciones de finales de julio sobre las clases de mayor tamaño (VIII y IX, 315 y $15-25 \mathrm{mg}$, respectivamente) que desaparecieron del tramo de estudio. Sin embargo, tras las precipitaciones, la estructura de la comunidad volvió a estabilizarse, de manera que tanto los valores de densidad, como los de biomasa fueron los más elevados de todo el periodo de estudio para cinco de las nueve clases de tamaño (I, V, VII, VIII y IX).

\section{Estructura de tamaño de la comunidad de macroinvertebrados acuáticos por hábitat}

Cuando se analizan los datos de densidad y biomasa obtenidos en cada uno de los hábitats prospectados (Fig. 6), se observa que las mayores densidades de organismos aparecen asociadas a los hábitats HT2 (7273 ind $\left./ \mathrm{m}^{2}, \mathrm{n}=15\right) \mathrm{y}$ HT12 (6104 ind/ $\mathrm{m}^{2}, \mathrm{n}=3$ ), siendo el hábitat HT4 el que alberga menor densidad de individuos (755 ind $\left./ \mathrm{m}^{2}, \mathrm{n}=6\right)$. En términos de biomasa, es el hábitat HT11 $\left(0.91 \mathrm{~g} \mathrm{PS} / \mathrm{m}^{2}\right)$ el que soporta mayor biomasa, seguido por el HT12 (0.64 g $\left.\mathrm{PS} / \mathrm{m}^{2}, \mathrm{n}=12\right)$. En general, los hábitats de corriente presentan mayor biomasa $(2.41 \mathrm{~g}$ $\mathrm{PS} / \mathrm{m}^{2}$ ) que los profundos $\left(1.16 \mathrm{~g} \mathrm{PS} / \mathrm{m}^{2}\right) \mathrm{y}$ someros $\left(0.5 \mathrm{~g} \mathrm{PS} / \mathrm{m}^{2}\right)$.

Con relación a la densidad de organismos, la mayor parte de los hábitats, están dominados por individuos de la clase de tamaño II, como ocurre en el espectro general.

Las clases de tamaño I y II, formadas fundamentalmente por larvas pequeñas de quironomidos y ceratopogonidos, aparecen asociadas a los hábitats HT2 y HT12. En los hábitats HT3 y HT10 se detectaron los valores de densidad y biomasa más elevados de las clases de tamaño III y IV, en el primer caso debido al heteróptero Micronecta scholtzi, y en el segundo al coleóptero Ochthebius tacapasensis, a los oligoquetos mayores de $2 \mathrm{~mm}$ y a los ceratopogonidos mayores de $4 \mathrm{~mm}$. Las clases de tamaño V y VI presentan densidades elevadas en los hábitats de corriente HT11, HT1 y HT8 debido a 


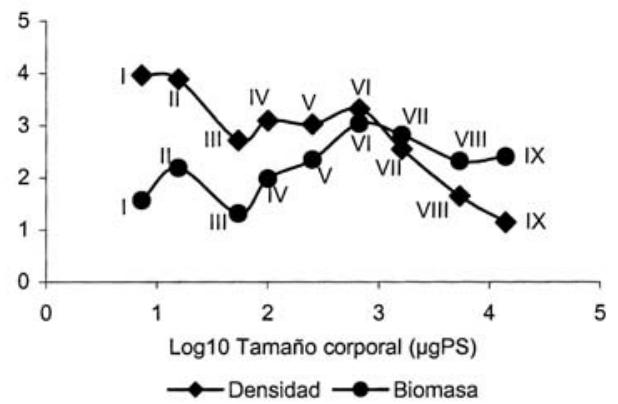

3-08-99

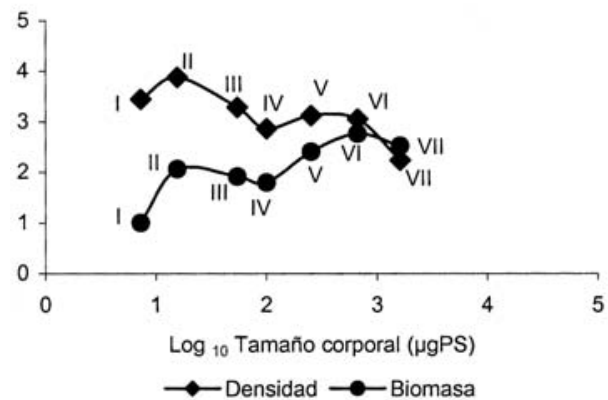

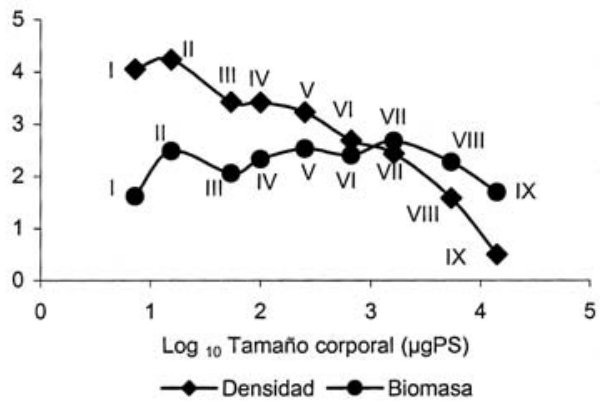

17-08-99

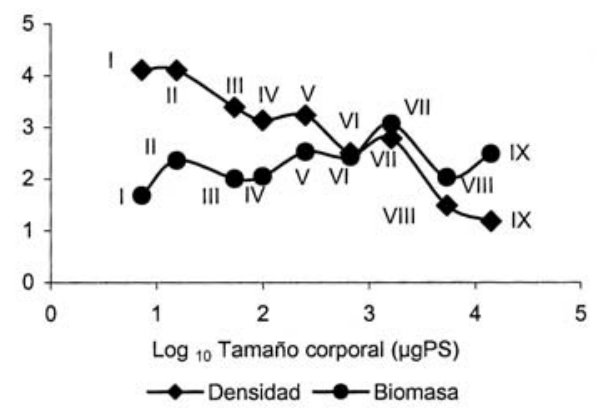

1-09-99



Figura 5. Espectro de tamaños de la comunidad de macroinvertebrados acuáticos del Río Chícamo para cada una de las prospecciones realizadas. La densidad está expresada en ind $/ \mathrm{m}^{2}$ y la biomasa en $\mathrm{mg} \mathrm{PS} / \mathrm{m}^{2}$. Size spectra of the macroinvertebrate community in the Chicamo stream for each sampling date. Density is expressed as ind $/ \mathrm{m}^{2}$, and biomass as $\mathrm{mg} \mathrm{DW} / \mathrm{m}^{2}$.

Echinogammarus veneris. En los hábitats, HT11, HT4 y HT12 destacan las abundancias de los coleópteros Nebriporus baeticus, Berosus hispanicus, en los dos primeros y de $N$. baeticus, Enochrus falcarius, y del efemeróptero Cloeon inscriptum en el último.
En general, las clases de tamaño que incluyen a los individuos más grandes están asociadas a hábitats con presencia de vegetación terrestre y mixta (HT6, HT10, HT12), a la que pertenece del odonato Orthetrum brunneum, típico habitante de hábitats con densa vegetación. En 


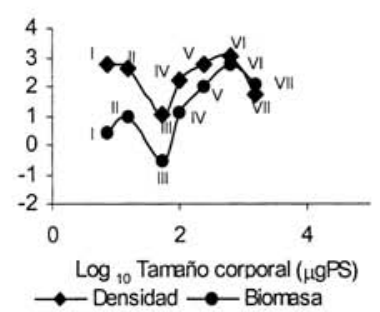

HT5

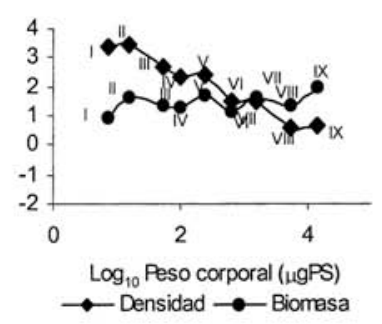

HT9

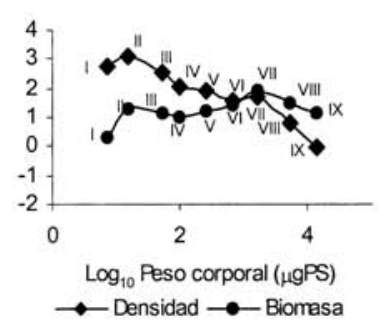



$\rightarrow$ Densidad $\multimap-$ Biomasa

HT6
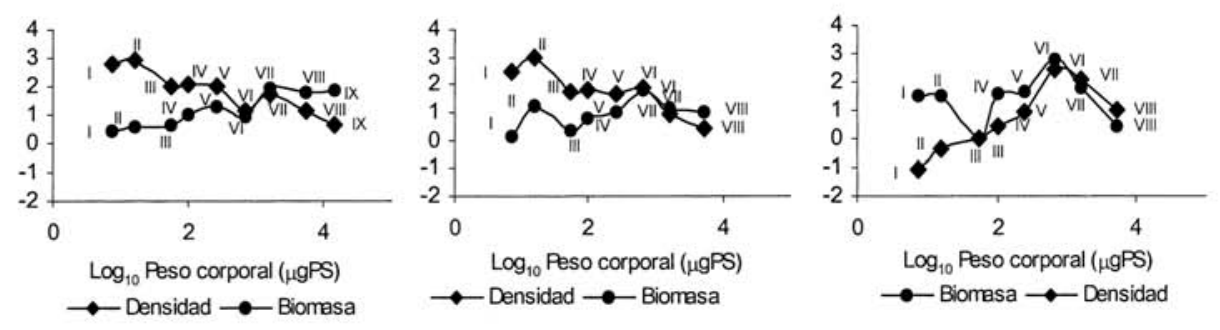

HT10
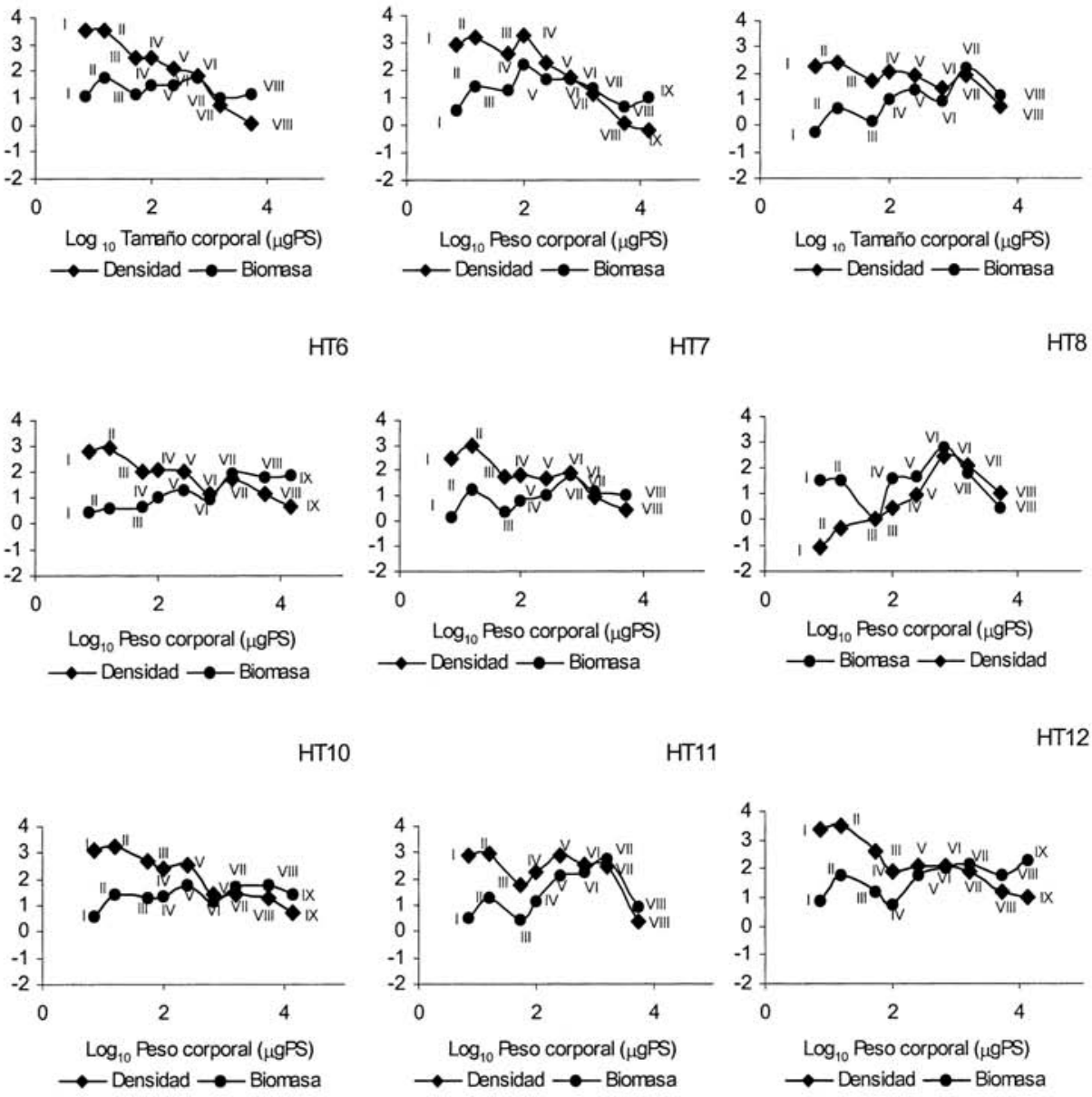

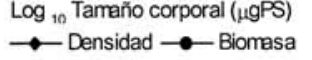

HT8

Figura 6. Espectro de tamaño de la comunidad de macroinvertebrados acuáticos del río Chícamo en cada uno de los hábitats en el

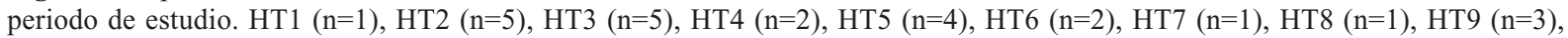
HT10 $(\mathrm{n}=3)$, HT11 $(\mathrm{n}=4)$, HT12 $(\mathrm{n}=1)$. La densidad está expresada en ind $/ \mathrm{m}^{2}$ y la biomasa en $\mathrm{mg}$ PS/m². Size spectra of the aquatic macroinvertebrate community in the Chicago stream in each one of the habitats along time. HT1 $(n=1), H T 2(n=5), H T 3(n=5)$, HT4 $(n=2), H T 5(n=4), H T 6(n=2), H T 7(n=1), H T 8(n=1), H T 9(n=3), H T 10(n=3), H T 11(n=4)$, HT12 ( $n=1)$. Density expressed as ind $/ \mathrm{m}^{2}$, and biomass as $m g \mathrm{DW} / \mathrm{m}^{2}$.

relación a la biomasa, existe mucha mayor variabilidad (Fig. 6).

\section{Estructura de tamaño de la comunidad de macroinvertebrados acuáticos y la heterogeneidad espacio-temporal}

Con el fin de analizar el efecto del estiaje y de las lluvias registradas durante el periodo de estudio (variable fecha), y la heterogeneidad espacial (variable hábitat), en el espectro de tamaño de la comunidad de macroinvertebrados acuáticos del río Chícamo, se llevó a cabo un GLM (Modelo Lineal General). Como puede observarse en la Tabla 6, los términos lineal y cuadrático del peso, explican por si solos el $59 \%$ de la varianza de la densidad de organismos por clase de tamaño. La varianza explicada, se incrementa en un $2.5 \%$, cuando se añade al modelo la fecha de muestreo y el hábitat en el que se tomaron las 
Tabla 6. Resultados del análisis del Modelo Lineal General Secuencial (GLM) sobre la matriz de densidad de macroinvertebrados por clase de tamaño durante el periodo de estudio. Todas las variables resultaron significativas para $\mathrm{p}<0.05(\mathrm{n}=621)$, a excepción de la interacción Hábitat x Peso. El error cuadrado medio (MSE) y el coeficiente de determinación $\left(\mathrm{R}^{2}\right)$ se muestran para cada uno de los pasos del análisis. Results of the Sequential General Linear Model (GLM) on the matrix density of the macroinvertebrates per size class during the study period. All variables were significant for $p<0.05$ ( $n=621$ ), except for the habitat $x$ weight interaction. Mean square error (MSE) and $R^{2}$ are shown for each one of the steps in the analysis.

\begin{tabular}{lll}
\hline Variables independientes & MSE & $\mathbf{R}^{\mathbf{2}}$ \\
\hline $\log _{10}($ peso $), \log _{10}(\text { peso })^{2}$ & 0.288 & 0.590 \\
Fecha & 0.283 & 0.598 \\
Hábitat & 0.271 & 0.615 \\
Hábitat x Fecha & 0.260 & 0.631 \\
Fecha x Peso & 0.254 & 0.639 \\
Hábitat x Fecha x Peso & 0.250 & 0.645 \\
\hline
\end{tabular}

muestras ( $\mathrm{R}^{2}$ aumenta de 0.590 a 0.615 , Tabla 6), y aumenta en un $3 \%$ más, cuando se añaden las interacciones de dos y tres factores $\left(\mathrm{R}^{2}\right.$ aumenta de 0.615-0.645, Tabla 6).

El análisis indica que las variaciones espaciales adquieren mayor relevancia que las temporales en la distribución de los organismos por clases de tamaño y que la interacción de los tres factores analizados: peso $\mathrm{x}$ hábitat $\mathrm{x}$ fecha, al ser positiva y significativa $(\mathrm{p}<0.05)$, pone de manifiesto una concentración de los organismos de mayor tamaño, al menos, en un hábitat y una fecha concreta.

\section{Estructura de tamaño de la comunidad de macroinvertebrados acuáticos y las variables ambientales}

Un segundo análisis GLM se realizó para determinar el efecto que la textura del sustrato, la biomasa de perifiton y la concentración de PRS en cada uno de los hábitat analizados, tienen sobre la densidad y distribución, en clases de tamaño de los taxones que componen la comunidad de macroinvertebrados acuáticos del río Chícamo.

No se han encontrado correlaciones entre ninguno de los parámetros analizados y los valores de densidad de organismos por clase de tamaño, lo que parece indicar que tanto la densidad como la distribución de los organismos por clase de tamaño, presentan valores similares en hábitats con diferentes tipo de sustrato, biomasa de perifiton y concentración de fósforo.

\section{DISCUSIÓN}

La comunidad de macroinvertebrados acuáticos del río Chícamo, está claramente dominada por los dípteros, de manera que para todas las fechas analizadas y en todos los hábitats muestreados, son las larvas de las familias Ceratopogonidae y Chironomidae, en ese orden, las que presentan mayor número de individuos. Más concretamente son los comprendidos entre 1-4 mm de longitud corporal, los que aportan en número, el $70 \%$ de los organismos recogidos. Este elevado número de individuos supone únicamente el $8.24 \%$ de la biomasa total medida en el tramo de estudio, siendo los insectos de mayor tamaño los que tienden a ser menos abundantes, aunque son los que mayores aportaciones realizan a la biomasa total $(69 \%)$. Los coleópteros y en especial Nebrioporus baeticus (valor medio $=0.55 \mathrm{~g} \mathrm{PS} / \mathrm{m}^{2}$ ) es el responsable del $24 \%$ de la biomasa total medida en el tramo de estudio. Morin \& Nadon (1991) comentan que entre el $82-98 \%$ de la biomasa total corresponde a los individuos con tamaños corporales $>2 \mathrm{~mm}$, mientras que las aportaciones de los organismos de tamaños inferiores son prácticamente despreciables. En una revisión realizada por Huryn \& Wallace (2000) sobre 57 estudios que estiman la biomasa de comunidades de macroinvertebrados acuáticos incluida la meiofauna, revela que en el 68\% de los casos, la biomasa es menor de $5 \mathrm{~g} \mathrm{PS} / \mathrm{m}^{2}$ y que el $81 \%$ es menor de $10 \mathrm{~g} \mathrm{PS} / \mathrm{m}^{2}$. En el tramo de estudio, la biomasa de la fracción de la comunidad analizada muestra unos valores de biomasa totales de $11.55 \mathrm{~g} \mathrm{PS} / \mathrm{m}^{2}$, que equivalen a $2.3 \mathrm{~g} \mathrm{PS} / \mathrm{m}^{2} \mathrm{de}$ media durante las cinco semanas de muestreo.

Si bien el resultado obtenido parece bajo (Huryn \& Wallace, 2000), el estudio está realizado en una única época del año, en la que, aun- 
que en el río Chícamo la densidad de organismos alcanza valores muy elevados, no son los máximos anuales (Guerrero, 1996) sino que son los individuos de pequeño tamaño los que asumen los mayores incrementos, y los que presen$\tan$ los menores valores de biomasa.

Cuando se analiza la composición específica de la comunidad, comparándola con los datos obtenidos a lo largo de un ciclo hidrológico completo (Guerrero, 1996), se observa una simplificación durante el estiaje, de manera que especies como los heterópteros Nepa cinerea y Naucoris maculatus, los odonatos Crocothemis erythraea y Anax parthenope, o las diferentes especies de moluscos (fundamentalmente Melanopsis praemorsa) prácticamente han desaparecido del tramo de estudio. Las razones de estas bajas densidades, aunque pueden ser de naturaleza variada, probablemente son consecuencia directa de los procesos asociados a la disminución drástica de la superficie inundada. La pérdida de hábitats de corriente así como el aumento, durante el estiaje, de sales en el agua, determina que especies como los moluscos Theodoxus fluviatilis y Melanopsis praemorsa lleguen a desaparecer de la comunidad, y que la familia Chironomidae disminuya su presencia, como ocurre en éste y otros trabajos (Muñoz \& Prat, 1994). Además, en un estudio llevado a cabo en el año 1996 en el mismo tramo del río Chícamo, se encuentra una situación similar ya que $A$. parthenope, C. erythraea y N. cinerea desaparecieron de la comunidad durante el estiaje (Guerrero, 1996). Otras especies como Caenis luctuosa, con valores muy elevados de densidad y producción en el tramo de estudio,(1233.72 ind $/ \mathrm{m}^{2}, 8.158 \mathrm{~g} \mathrm{~m}^{-2}$ año ${ }^{-1}$, Perán et al., 1999), aunque no ha desaparecido, puesto que se localizó aguas-abajo del tramo de estudio en una poza con mayor profundidad, sí lo hizo en el tramo prospectado. Por último destacar, que las larvas de odonatos recogidas en el presente trabajo, son muy pequeñas en longitud corporal, en comparación con las tallas finales que alcanzan en otras épocas del año (p.e. larvas de Anax parthenope: $8 \mathrm{~mm}$, tamaño máximo alcanzado por la larva: 50 $\mathrm{mm})$. Esta simplificación de la comunidad afecta por tanto, a los organismos de mayor tamaño con presencia en el río Chícamo, lo que implica que durante este periodo del ciclo hidrológico los valores de biomasa estimados probablemente sean inferiores a los que podrían registrarse si el estudio se ampliara a un ciclo hidrológico completo.

Cuándo se intenta establecer comparaciones con otros sistemas, la comunidad de macroinvertebrados del río Chícamo, está constituida por especies con tasas de crecimiento rápidas, ciclos de vida cortos y tamaños corporales en los estadios finales de desarrollo, generalmente inferiores a los mostrados en la literatura como adaptación a las perturbaciones (Gray, 1981; Ortega et al., 1991). Esto implica en general, valores de biomasa menores a los detectados en sistemas más estables en los que las comunidades están compuestas por especies con ciclos de vida largos, que les permiten alcanzar tamaños corporales superiores (Jackson \& Fisher 1986; Gray, 1989).

Los resultados de biomasa obtenidos son muy inferiores a los detectados en otro sistema de características similares, en cuanto a régimen de perturbaciones, estructura trófica y ciclos de vida de las especies, como es el caso de Sycamore Creek, en Arizona ( $2.98 \mathrm{~g} / \mathrm{m}^{2}$, sólo para el grupo trófico de los colectores, Fisher \& Gray 1983). Sin embargo, en los ríos de las praderas americanas de características parecidas a las del río Chícamo, en cuanto a su régimen estacional de perturbaciones (avenidas y estiaje), autotrofía, estructura trófica y temperaturas medias (Gray \& Johnson, 1988; Dodds et al., 1996; Miller \& Golladay, 1996; Gray, 1997), el valor de biomasa obtenido para un ciclo hidrológico completo es de 2.3 g PSLC $/ \mathrm{m}^{2}$ (Stagliano \& Whiles, 2002) similar a los $2 \mathrm{~g}$ PSLC $/ \mathrm{m}^{2}$ del río Chícamo.

Los depredadores aportan el $58.4 \%$ de la biomasa muy superior a la del resto de grupos funcionales con representantes en el tramo de estudio: colectores-raspadores $(39.9 \%)$ y colectores-filtradores (1.7\%). Estos datos contradicen lo predicho por el RCC (River Continuum Concept, Vannote et al 1980; Cummins, 1988) en cuanto a que la apor- 
tación de los depredadores es de aproximadamente el $10 \%$, independientemente del orden del río estudiado, pero apoyan los resultados obtenidos en diferentes trabajos, como el de Lugthart \& Wallace (1992) quienes establecen, para ríos de cabecera, una contribución de los depredadores superior al 10\% (33-45\%) o el de Smock et al. (1985) que aporta valores cercanos al $30 \%$ en condiciones de ausencia de flujo.

En el río Chícamo la densidad de los depredadores (exceptuando a las posibles especies depredadoras pertenecientes a las familias Chironomidae y Ceratopogonidae), aumenta a medida que el proceso de desecación avanza sobre el sistema, lo cual va acompañado por una disminución de las presas potenciales, fundamentalmente los colectores-raspadores. Este incremento probablemente este relacionado con la ausencia de depredadores de grupos tróficos superiores en el sistema (Schoefield et al., 1988), o como apuntan otros autores para ríos intermitentes (Hynes, 1975; Abell 1984; Boulton \& Lake, 1992; Boulton, 2003) o para charcas temporales (Lake et al., 1989), se trate de uno de los efectos del avance del proceso de desecación sobre el sistema, ya que los depredadores hacen coincidir sus periodos reproductores con la época del año en la que existe una mayor densidad de presas (Wiggins et al., 1980; Boulton \& Lake, 1992). Como se observa en el río Chicamo, la disminución de la densidad de las posibles presas, parece confirmar el incremento de la presión depredadora (Canton et al., 1984), que en este caso sería una consecuencia directa de la disminución de la lámina de agua: la concentración de organismos en aquellas partes del cauce que permanecen inundadas (Stanley et al., 1997; Lake, 2000).

La estructura de tamaño obtenida para la comunidad de macroinvertebrados acuáticos del río Chícamo, se aleja de las curvas unimodales típicas de las comunidades de organismos en sistemas lóticos (Morin \& Nadon, 1991; Bourassa \& Morin, 1995; Morin, 1997; Solimini et al., 2001). La forma de la curva pone de manifiesto la no inclusión de la meiofauna, que constituiria el extremo ini- cial de la curva obtenida. Este componente, podría llegar a ser muy abundante en el tramo de estudio, dadas sus condiciones leníticas y el predominio de sedimento fino, que son las condiciones idóneas para el desarrollo de estos organismos (Suren, 1992).

Además, en estos estudios, los tamaños de luz de malla utilizados varían entre $10 \mu \mathrm{m}$ empleados por Schtwinghamer (1981) y $63 \mu \mathrm{m}$ por Bourassa \& Morin (1995), de manera que probablemente se ha subestimado la densidad de los organismos pertenecientes a las clases de tamaño $<2 \mathrm{~mm}$, así como la fracción de la comunidad con longitudes corporales $<1 \mathrm{~mm}$. Además, la utilización de etanol como medio de conservación determinó, que las larvas de menor tamaño se encontraran en mal estado de conservación y no se pudieran incluir en el análisis.

Aunque existen relativamente pocos estudios sobre la distribución de tamaños de las comunidades de invertebrados en ríos, la información existente revela una regularidad importante, y por tanto un cierto grado de predecibilidad (Morin, 1997). La distribución de tamaños de los invertebrados acuáticos, es generalmente lognormal, con la moda situada en $1 \mu \mathrm{g}$ PS, que correspondería a individuos con tamaños corporales de $1 \mathrm{~mm}$ aproximadamente. Para la comunidad de macroinvertebrados acuáticos del río Chícamo la moda en el espectro general, se sitúa en $1.2 \mu \mathrm{g}$ PS aunque ésta corresponde a individuos con longitudes corporales entre 2 y 4 $\mathrm{mm}$. Sin embargo, los individuos con longitudes corporales entre 1-2 $\mathrm{mm}$ (mayoritarios en dos de las cinco prospecciones) presentan la moda en $0.9 \mu \mathrm{g}$ PS, datos que no se alejan de forma importante, de los obtenidos en los modelos predictivos. Esto parece confirmar por tanto, que las diferencias existentes entre la estructura de tamaño de la comunidad de macroinvertebrados acuáticos del río Chícamo y la obtenida en los trabajos mencionados anteriormente, radica en la inclusión de la meiofauna como parte fundamental de las comunidades de invertebrados que habitan en los ríos.

Tanto los efectos del estiaje como de las precipitaciones registradas en el periodo de estu- 
dio, parecen influir en la densidad de organismos y en su estructura de tamaño, lo que queda de manifiesto en los resultados obtenidos en el primer Modelo Lineal General (GLM) realizado. Dado que las correlaciones son positivas entre los tres factores, parece indicar la existencia de, al menos, un hábitat concreto y en una fecha concreta, en el que existe una mayor densidad y por tanto biomasa, de los organismos de mayor tamaño presentes en el río Chícamo. Aunque el tipo de análisis realizado no muestra en concreto de que hábitat se trata, el análisis de las matrices de datos, confirman que es el hábitat HT5 (de corriente con sustrato mixto y perifiton) en el que se concentra un mayor número de individuos correspondientes a las clases de tamaño VIII y IX durante las primeras semanas de muestreo. Esta posible interpretación se contrapone a otra alternativa, y es la desaparición de ambas clases de tamaño el 3/8/99, a consecuencia de las precipitaciones.

Aunque generalmente encontramos estructuras de tamaño completas en los hábitats que presentan mayor profundidad, (HT3, HT6, HT9 y HT12), los hábitats HT10 y HT5 (profundidad entre 3-0.5 cm) también presentan estructuras completas. Si a este hecho se añade el que los individuos de mayor tamaño presentan sus mayores densidades asociadas al hábitat HT5 al comienzo del periodo de estudio, y al HT10, junto con el HT12, al final del mismo, podemos considerar que la profundidad, que en principio podría haber actuado como factor limitante a la presencia de individuos de gran tamaño en determinados hábitats, no influye en su distribución. Esto se apoya en que en ambos tipos de hábitats se han encontrado ejemplares del odonato Orthetrum brunneum con una longitud corporal de $1.7 \mathrm{~cm}$, tamaño próximo al máximo que puede alcanzar la especie $(2 \mathrm{~cm})$.

La falta de correlaciones entre la densidad y la distribución de los organismos por clase de tamaño, en hábitats con diferente tipo de sustrato, biomasa de perifiton y concentración de fósforo, viene a confirmar lo obtenido por Bourassa \& Morin (1995), indicando que ni la producción primaria ni la secundaria, están en principio limitadas durante este periodo del ciclo hidrológico. Quizás porque durante el estiaje, las concentraciones de PRS se incrementan en el agua, debido a la descomposición del perifiton (VidalAbarca et al., 2000) y a las entradas por escorrentía tras las precipitaciones registradas en el tramo de estudio (Sánchez, com. per.).

El análisis de la estructura de tamaño de la comunidad de macroinvertebrados acuáticos del río Chícamo, es similar en todos los tipos de sustrato muestreados. Estos resultados, son similares a los obtenidos para otros sistemas lóticos (Morín \& Nadon, 1991; Bourassa \& Morín, 1995), para los que sin embargo, si existen diferencias significativas en los valores de densidad o abundancia en función de este parámetro. Probablemente el resultado obtenido en el presente estudio, esté relacionado con la existencia de un claro dominio de los hábitats someros a medida que avanza el proceso de desecación, recordando que las mayores densidades detectadas en el tramo de estudio, aparecen asociadas a este tipo de hábitats. Sin embargo, los hábitats de corriente, aunque muy numerosos al comienzo del estudio, sufrieron una reducción importante, tanto en número de manchas (5 manchas al final del estudio) como en superficie ocupada (reducción del 93\%), en el transcurrir de las semanas a consecuencia de la disminución del flujo.

\section{AGRADECIMIENTOS}

Maria Sánchez-Montoya e Isabel Hurtado compartieron el trabajo de campo y aportaron algunos datos para la elaboración de este trabajo. Magdalena Martínez participó en la tediosa tarea de separación de los organismos acuáticos. Los datos termopluviométricos han sido proporcionados por el Instituto Meteorológico del Sureste (Ministerio de Medio Ambiente). Los resultados de este estudio han sido financiados por el Proyecto REN2001-0644-C02-01/HID, Dirección General de Investigación, Ministerio de Ciencia y Tecnología. Dos lectores anónimos nos ayudaron a sintetizar mejor el trabajo. 


\section{BIBLIOGRAFÍA}

ABELL, D. L. 1984. Benthic invertebrates of some California intermittent streams. In: Vernal Pools and Intermittent Streams. S. Jain \& P. Moyle (eds.).: 46-60. University of California, Davis Institute of Ecology, Publication No. 28.

ALOI, J. E. 1990. Critical review of recent freshwater periphyton field methods. Can. J. Fish. Aquat. Sci., 47: 656-670.

A.P.H.A. 1985. Standard methods for the examination of water and waste water. $16^{\text {th }}$ ed. Amer. Public Health Assoc., Inc. Washington.

BASTOW, J. L., J. L. SABO, J. C. FINLAY \& M. E. POWER. 2002. A basal aquatic-terrestrial trophic link in rivers: algal subsides via shore-dewelling grasshoppers. Community Ecology on line: http: //link.springer.de/link/service/jo...00879/paper/s0 0442-002-0879-7ch100.html.

BOULTON, A. J. 2003. Parallels and contrasts in the effects of drought on stream macroinvertebrate assemblages. Freshwat. Biol., 48: 1173-1185.

BOULTON, A. J. \& S. LAKE. 1992. The ecology of two intermittent streams in Victoria, Australia. III. Temporal changes in faunal composition. Freshwat. Biol., 27: 123-138.

BOURASSA, N. \& A. MORIN. 1995. Relationships between size structure of invertebrate assemblages and trophy and substrate composition in streams. J. N. Am. Benthol. Soc., 14(3): 393-403.

BURGHERR, P. \& E.I. MEYER. 1997. Regression analysis of linear body dimensions vs. dry mass in stream macroinvertebrates. Arch. Hydrobiol., 139(1): 101-112.

CANTON, S. P., L. D. CLINE, R. A. SHORT \& J. V. WARD. 1984. The macroinvertebrates and fish of a Colorado stream during a period of fluctuating discharge. Freshwat. Biol., 14: 311-316.

CUMMINS, K. W. 1966. A review of stream ecology with special emphasis on organism-substrate relationships. Pymatuning Lab. Ecol. Special Publ., 4: 2-51.

CUMMINS, K.W. 1973. Trophic relations of aquatic insects. Ann. Rev. Entomol., 18: 183-206.

CUMMINS, K.W. 1988. The study of stream ecosystems: a functional review. In: Concepts of ecosystem ecology. L.R. Pomeroy \& J.J. Alberts (eds.).: 247-262. Springer-Verlag, New York.

CUMMINS, K. W. \& G. H. LAUFF. 1969. The influence of substrate particle size on the microdistribution of stream macrobenthos. Hydrobiologia, 34: 145-181.
DODDS, W. K., R. E. HUSTON, A. C. EICHEM, M. A. EVANS, D. A. GUDDER. K. M. FRITZ \& L. J. GRAY. 1996. The relationship of floods, drying, flow and light to primary production and producer biomass in a prairie stream. Hydrobiologia, 333: 151-159.

DOWNES, B. J., P. S. LAKE \& E. S. G. SCHREIBER. 1993. Spatial variation in the distribution of stream invertebrates: implications of patchiness for models of community organization. Freshwat. Biol., 30: 119-132.

EGGLISHAW, J. M. 1964. The distributional relationship between the bottom fauna and plant detritus in streams. J. Anim. Ecol., 33: 463-476.

FISHER, S. G. \& L. J. GRAY. 1983. Secondary production and organic matter processing by collector macroinvertebrates in a desert stream. Ecology, 64(5): 1217-1224.

GRAY, L. J. 1981. Species composition and life histories of aquatic insects in a lowland Sonoran Desert stream. Am. Midl. Nat., 106: 229-42.

GRAY, L. J. 1989. Emergence production and export of aquatic insects from a tallgrass prairie stream. Southwest. Nat., 34: 313-318.

GRAY, L. J. 1997. Organic matter dynamics in Kings Creek, Konza Prairie, Kansas, USA. In: Stream organic matter budgets. J. R. Webster \& J. L. Meyer (eds.).:50-54. J. N. Am. Benthol. Soc. 16.

GRAY, L. J. \& K. W. JOHNSON. 1988. Trophic structure of benthic macroinvertebrates in Kings Creek. Transactions of the Kansas Academy of Sciences, 91: 178-184.

GUERRERO, M. C. 1996. Los invertebrados acuáticos del río Chícamo (SE de España): variación espacio-temporal. Tesis de Licenciatura. Universidad de Murcia.

GUERRERO, M. C. 2002. Patrones ecológicos y respuesta de la comunidad de macroinvertebrados acuáticos al estiaje. El caso del río Chícamo (SE de España). Tesis Doctoral. Universidad de Murcia.

HANSON, J. M., E. E. PREPAS \& W. C. MACKAY. 1989. Size distribution of the macroinvertebrates community in a freshwater lake. Can. J. Fish. Aquat. Sci., 46:1510-1519.

HURYN, A. D. \& J. B. WALLACE. 1987. Producction and litter processing by crayfish in an Appalachian stream. Freshwat. Biol., 18: 277-286.

HURYN, A. D. \& J. B. WALLACE. 2000. Life history and production of stream insects. Annu. Rev. Entomol., 45: 83-110. 
HYNES, A. H. 1975. Annual cycles of macro-invertebrates of a river in southern Ghana. Freshwat. Biol., 5: 71-83.

HYNES, A. H. 1982. Allometric constraints and variables of reproductive effort in brachyran crabs. Mar. Biol., 69: 309-320.

JACKSON, J. K. \& S. G. FISHER, 1986. Secondary production, emergence, and export of aquatic insects of a Sonoran Desert stream. Ecology, 67: 629-638.

LAKE, P. S., I. A. E. BAYLY \& D. W. MORTON. 1989. The phenology of a temporary pond in western Victoria, Australia, with special reference to invertebrate succession. Arch. Hydrobiol., 115: 171-202.

LAKE, P. S. 2000. Disturbance, patchiness, and diversity in streams. J. N. Am. Benthol. Soc., 19(4): 573-592.

LUGTHART, G. J. \& J. B. WALLACE. 1992. Effects of disturbance on benthic functional structure and production in mountain streams. J.N. Am. Benthol. Soc., 11(2): 138-164.

MARTÍNEZ, B., J. VELASCO, M. L. SUÁREZ \& M. R. VIDAL-ABARCA. 1998. Benthic organic matter dynamics in an intermittent stream in South-East Spain. Arch. Hydrobiol. , 141(3): 303 320.

MILLER, A. M. \& S. W. GOLLADAY. 1996. Effects of spates and drying on macroinvertebrate assemblages of an intermittent and a perennial prairie streams. J. N. Am. Benthol. Soc., 15(4): 670-689.

MORIN, A. 1991. Intensity and importance of abiotic control and inferred competition on biomass distribution patterns of Simuliidae and Hydropsychidae in southern Quebec streams. J. N. Am. Benthol. Soc., 10(4): 388-403.

MORIN, A. 1997. Empirical models predicting population abundance and productivity in lotic systems. J. N. Am. Benthol. Soc., 16(2): 319-337.

MORIN, A. \& D. NADON. 1991. Size distribution of epilithic lotic invertebrates and implications for community metabolism. J. N. Am. Benthol. Soc., 10(3): 300-308.

MORIN, A. \& P. DUMONT. 1994. A simple model to estimate growth rate of lotic insects larvae and its value for estimating population and community production. J. N. Am. Benthol. Soc., 13(1): 357367.

MORIN, A.., M. A. RODRIGUEZ \& D. NADON. 1995. Temporal and spatial variation in the biomass spectrum of benthic invertebrates in streams: an application of thin-plate splines and relative warp analysis. Can. J. Fish. Aquat. Sci., 52: 18811892.

MUÑOZ, I. \& N. PRAT. 1994. Macroinvertebrate community in the lower Ebro River (NE Spain). Hydrobiologia, 286: 65-78.

NETER, J. M., H. HUTNER, C. J. NACHTSHEIM \& W. WASSEMAN. 1996. Applied linear statistical models. Irwin, Chicago.

ORTEGA, M., M. L. SUÁREZ, M. R. VIDALABARCA \& L. RAMÍREZ-DÍAZ. 1991. Aspectos dinámicos de la composición y estructura de la comunidad de invertebrados acuáticos de la Rambla del Moro después de una riada (cuenca del río Segura: SE de España). Limnetica, 7: 1124.

PALMER, M. A. \& N. L. POFF. 1997. The influence of environmental heterogeneity on patterns and processes in streams. J. N. Am. Benthol. Soc., 16(1): 169-173.

PALMER, M. A., C. C. HAKENKAMP \& K. NELSON-BAKER. 1997. Ecological heterogeneity in streams: why variance matters. $J . N$. Am. Benthol. Soc., 16(1): 189-202.

PERÁN, A., J. VELASCO \& A. MILLÁN. 1999. Life cycle and secondary production of Caenis luctuosa (Ephemeroptera) in a semiarid stream (Southeast Spain). Hydrobiologia, 400: 187-194.

POFF, N. L., M. A. PALMER, P. L. ANGERMEIER, R. L. VADAS, C. C. HAKENKAMP, A. BELY, P. ARENSBURGER \& P. MARTIN. 1993. Size structure of the metazoan community in a piedmont stream. Oecologia, 95: 202-209.

SCHOEFIELD, K., C. R. TOWNSEND \& A. G. HILDREW. 1988. Predation and prey community of a headwater stream. Freshwat. Biol., 20: 85-95.

SCHWINGHAMER, P. 1981. Characteristic size distribution of integral benthic communities. Can. J. Fish. Aquat. Sci., 38: 1255-1263.

SHELDON, R. W., A. PRAKASH \& W. H. SUTCLIFFE. 1972. The size distribution of particles in the ocean. Limnol. Oceanogr., 17: 327-340.

SHELDON, A. L. \& R. A. HAICK. 1981. Habitat selection and association of stream insects: a multivariate analysis. Freshwat. Biol., 11: 395-403.

SMOCK, L. A, E. GILINSKY \& D. L. STONEBURNER. 1985. Macroinvertebrates production in southeastern United States blackwater streams. Ecology, 66: 1491-1503.

SOLIMINI, A. G., A. BENVENUTI, R. D'OLIMPIO, M. DE CICCO \& G. CARCHINI. 
2001. Size structure of benthic invertebrates assemblages in a Mediterranean river. J. N. Am. Benthol. Soc., 20(3): 421-431.

SPRULES, W. G., J. M. CASSELMAN \& B. J. SHUTER. 1983. Size distribution of pelagic particles in lakes. Can. J. Fish. Aquat. Sci., 40: 17611769.

STAGLIANO, D. M. \& M. R.WHILES. 2002. Macroinvertebrate production and trophic structure in a tallgrass prairie headwater stream. J. N. Am. Benthol Soc., 21: 97-113.

STANLEY, E. H., S. G. FISHER \& N. B. GRIMM, 1997. Ecosystem expansion and contraction in streams. BioScience, 47(7): 427-435.

STRAYER, D., 1986. The size structure of a lacustrine zoobenthic community. Oecologia, 69: 513516.

STRAYER, D., 1991. Perspectives on the size structure of lacustrine zoobenthos, its causes, and its consecuences. J. N. Am. Benthol. Soc., 10(2): 210-221.

STURGES, H., 1926. The choice of a class-interval. J. Amer. Statist. Asoc., 21: 65-66.

SUÁREZ, M. L. \& M. R. VIDAL-ABARCA, 2000. Metabolism of a semi-arid stream of south-east Spain. Verh. Internat. Verein. Limnol., 27: 756-761.

SUREN, A. M., 1992. Meiofaunal communities associated with bryophytes and gravels in shaded and unshaded alpine streams in New Zealand. $N$. Z. J. Mar. Freshwat. Res., 26: 115-125.
TACHET, H., P. RICHOUX, M. BOURNAUD \& P. USSEGLIO-POLATERA. 2000. Invertébrés d'eau douce: systématique, biologie, écologie. CNRS Éditions. Paris.

VANNOTE, R. L., G. W. MINSHALL, K. W. CUMMINS, J. R. SEDELL \& C. E. CUSHING. 1980. The river continuum concept. Can. J. Fish. Aquat. Sci., 37: 130-137.

VELASCO, J., A. MILLÁN, M. R.VIDALABARCA, M. L. SUÁREZ, C. GUERRERO, \& M. ORTEGA. 2003. Macrophytic, epipelic and epilithic primary production in a semiarid Mediterranean stream. Freshwat. Biol., 48: 14081420.

VIDAL-ABARCA, M. R., M. L. SUÁREZ, J.L. MORENO, R. GÓMEZ \& I. SÁNCHEZ. 2000. Hidroquímica de un río de características semiáridas (río Chícamo; Murcia). Análisis espacio-temporal. Limnetica, 18: 57-73.

WALLACE, J. B. \& R. W. MERRIT. 1980. Filter feeding ecology of aquatic insects. Ann. Rev. Entomol., 25: 103-132.

WARWICK, R. M. \& I. R. JOINT. 1987. The size distribution of organisms in the Celtic Sea: from bacteria to metazoa. Oecologia, 73: 185-191.

WIGGINS, G. B., R. J. MACKAY \& I. M. SMITH, 1980. Evolutionary and ecological strategies of animals in annual temporary pools. Arch. Hydrobiol. Supplement, 58: 97-206. 